\title{
LUMINESCENCE OF CERIUM DOPED YTTRIUM ALUMINUM GARNET CERAMICS SYNTHESIZED IN THE FIELD OF RADIATION FLUX
}

\author{
Alpyssova G.K. ${ }^{1,2}$, Lisitsyn V.M. ${ }^{3}$, Karipbayev Zh.T. ${ }^{2}$, Golkovski M.G. ${ }^{4}$ \\ 1,2Academician E.A.Buketov Karaganda University, Karaganda, Kazakhstan, gulnur-0909@mail.ru \\ ${ }^{2}$ L.N. Gumilyov Eurasian National University, Nur-Sultan, Kazakhstan \\ ${ }^{3}$ National Research Tomsk Polytechnic University, Tomsk, Russia \\ ${ }^{4}$ Budker Institute of Nuclear Physics of the Siberian Branch of the RAS, Novosibirsk, Russia
}

\begin{abstract}
The paper presents the results of ceramic samples' dependence of luminescent properties on their position in the crucible during irradiation. For the synthesis, a blend of powders of aluminum oxide, yttrium oxide and cerium oxide was prepared. The ratio of oxides in the charge corresponded to stoichiometric. The particles of the oxide powders had a size of about 1 micron and less. Synthesis was carried out on gas pedal electron accelerator at Institute of Nuclear Physics SB RAS (Novosibirsk). Luminescent properties were measured on spectrofluorimeter SM 2203 "SOLAR" under excitation by monochromatic radiation at $450 \mathrm{~nm}$. Two types of samples differing in the value of bulk density and the prehistory of yttrium oxide were investigated.
\end{abstract}

Keywords: white LEDs, yttrium-aluminum garnet, phosphor, ceramics, synthesis in the radiation field.

\section{Introduction}

Synthesis on the basis of yttrium-aluminum garnet (YAG) materials of different morphologies (single crystals, powders, ceramics, films) is carried out using different technologies [1-8]. Exposure to powerful radiation streams can solve an important problem: increasing the reactivity of reagents to produce new materials and the efficiency of the synthesis of refractory materials [9-12]. High temperatures are used, preliminary preparation of mixtures of different compositions, addition of additional materials to facilitate the synthesis processes, etc. Obviously, this affects the reproducibility of the synthesis results. In addition, the complexity of the synthesis makes it difficult to optimize the technology in order to achieve the maximum values of luminescence characteristics.

In this work, the radiation synthesis of ceramics was carried out by scanning a powerful beam of electrons over the surface of the charge in the crucible. The scanning was carried out in such a way that the absorbed energy at each point of the surface was the same. The number, shape, and position of the samples in the crucible after synthesis in each experiment with the same initial charge doesn't depend on the direction of the beam movement. However, there is a tendency for differences in the shape and size of the samples obtained at the beginning and end of synthesis. The beam scans in the transverse direction of the crucible and shifts with each step from the initial position in the crucible to the final position. At a scanning speed of 1 $\mathrm{cm} / \mathrm{s}$, the movement of the beam in the transverse direction of the crucible takes $4 \mathrm{~s}$. In total the whole irradiation cycle from the beginning to the end of the crucible takes 36s [13]. At the beginning of the experiment the crucible has an ambient room temperature, which in the room is $20-25^{\circ} \mathrm{C}$. During the irradiation process, at a scanning speed of $1 \mathrm{~cm} / \mathrm{s}$, energy equal to (at a power density of $20 \mathrm{~kW} / \mathrm{cm}^{2}$ ) 20 $\mathrm{kJ} / \mathrm{cm} 2$ is transferred to the charge. Immediately upon interaction, $99 \%$ of the energy is spent on transferring electrons from valence to conduction zones [14-16].

It takes energy equal to 2-3 Eg to create one electron-hole pair in YAG. Bandgap width in YAG is 6.5 $\mathrm{eV}$, in metal oxides used for synthesis - YAG $\left(\mathrm{Y}_{2} \mathrm{O}_{3}, \mathrm{Al}_{2} \mathrm{O}_{3}\right)$ - about $8 \mathrm{eV}$. Relaxation of electrons and holes from states in zones, in which they appeared under influence of electron flux, to corresponding bottom of conduction zone for electrons and ceiling of valence zone for holes takes time less than 10-15s. During relaxation of each pair energy equal to $1-2 \mathrm{Eg}$ is released. Therefore, about $0.5-0.7$ energy of absorbed radiation is spent on heating of charge and forming ceramics. With an exposure dose of $20 \mathrm{~kJ} / \mathrm{cm}^{2}$ and a run depth in the charge equal to $0.6 \mathrm{~cm}, 15$ to $20 \mathrm{~kJ} / \mathrm{cm}^{3}$ is spent on heating. This is a lot of energy, which leads to heating of the charge and, consequently, the crucible. As the electron beam moves during scanning, the temperature of crucible and charge in the place of irradiation increases, the conditions of ceramic formation change. In works [17-22] the possibility of radiation synthesis of luminescent ceramics based on YAG was 
shown. For the synthesis, a charge of Al, Y oxides of stoichiometric composition with different contents of $\mathrm{Ce}, \mathrm{Gd}$ was prepared. Under the action of a powerful flux of electrons from the charge, ceramics with properties characteristic of YAG luminophores was formed.

\section{Experimental technique}

The object of the study is ceramics obtained by radiation synthesis. Ceramics were synthesized using the gas pedal ELV-6 at the INP of the Siberian Branch of the Russian Academy of Sciences (Novosibirsk). The electron gas pedal allows to generate electron fluxes with energies of $1.4 \mathrm{MeV}$ and power up to $100 \mathrm{~kW}$ (beam current $100 \mathrm{~mA}$ ) in steady-state mode. Beam power could be varied by varying the emitter current. Luminescence properties of ceramic samples were investigated depending on their position in the crucible during irradiation.

\section{Results and discussions}

The experiments were performed as follows. After synthesis, the crucible with the samples was cooled. The state of the synthesis result was photographed: the location of the samples in the crucible (Figure 1). Each sample was assigned a number. The numbering was carried out according to the location of the samples in the crucible from left to right, from top to bottom along the movement of the beam. Photographs of the ceramic samples synthesized using a power density of $20 \mathrm{~kW} / \mathrm{cm} 2$ and their appearance after crushing in packages are shown in Figure 1. The samples of 56 and 59 series were chosen for the research. The initial charge compositions for the synthesis of these samples were the same, but differed in the background of the yttrium oxide used. The synthesis was performed under the same irradiation conditions. The luminescence spectra of the powders were measured with a spectrofluorimeter SM 2203 made by SOLAR under excitation by monochromatic radiation at $450 \mathrm{~nm}$.

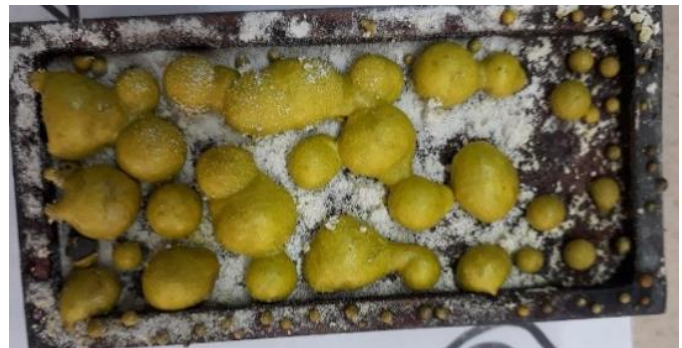

a)

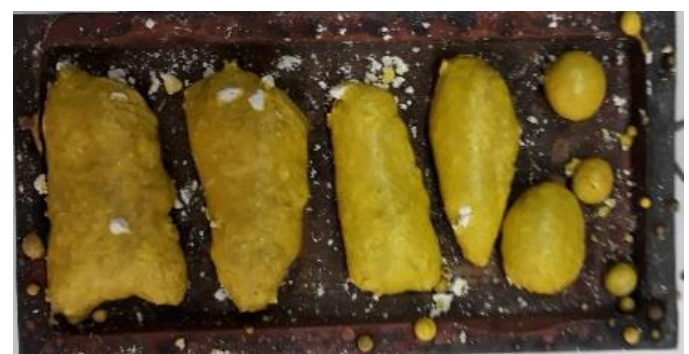

b)

Fig.1. Photos of 56 (a) and 59 (b) series samples in the crucible.

For luminescence measurements, samples were mechanically crushed and poured into plastic bags transparent in the spectral region of 350 to $800 \mathrm{~nm}$. To obtain reliable information about the characteristics of the spectra, the measurements were performed as follows. The luminescence spectra of each sample were measured 10 times. Before each measurement, the bag with the powder was shaken in order to mix the particles in the bag. Figure 2 shows, for example, the non-normalized luminescence spectra of samples 561and 56-13 in order to clearly show their similarity. In all measurements, the luminescence intensities were different.

This is due to the fact that we do not know the distribution of the powder in the excitation field and the emission in the excitation field. But this could only affect the intensity, but not the spectral characteristics of the luminescence, which is the subject of the research. The average values of the positions and half-widths of the bands and the values of measurement errors are presented in Table 1. When processing the measurement results, the outliers due to unknown causes were highlighted. Accordingly, the outliers were not taken into account when calculating the error.

A total of 13 samples were formed in crucible 56. Each sample was measured 10 times. Figure 3 (a,b) also shows diagrams of the dependence of the fringe position (a) and the half-width of the fringes (b) on the arrangement in the crucible. The red horizontal lines show the limiting measured values of the fringe position and the half-width of the fringes. The crucible average of the maximum band position is 555.8. The variation of the fringe position across the crucible is within 2-4 $\mathrm{nm}$. The variation of band half-widths is $0.01-0.02 \mathrm{eV}$. 


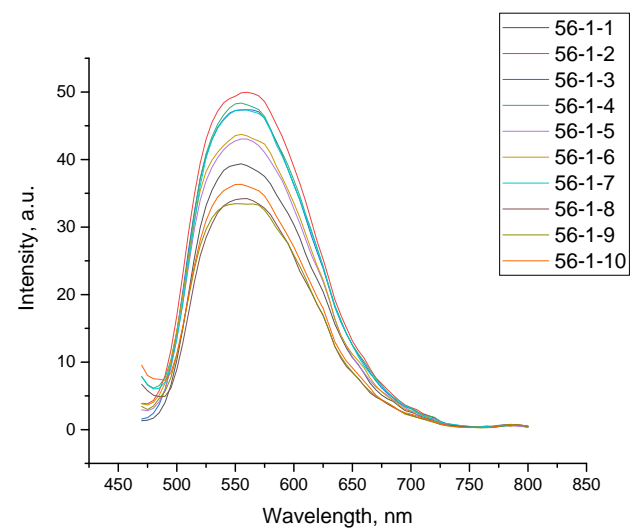

a)

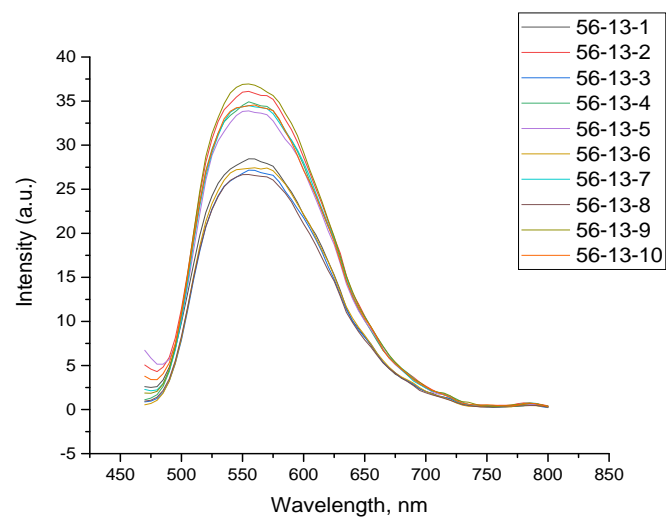

b)

Fig. 2. Luminescence spectra of samples from series: a) 56-1; b) 56-13

Table 1. Mean values of fringe positions and half-widths and values of measurement errors

\begin{tabular}{|c|c|c|}
\hline Sample name & $\boldsymbol{\lambda}_{\boldsymbol{s}}, \mathbf{n m}$ & $\boldsymbol{\Delta E}, \mathbf{e V}$ \\
\hline $56-1$ & $556.7 \pm 0.8$ & $0.455 \pm 0.01$ \\
\hline $56-2$ & $556.6 \pm 0.9$ & $0.455 \pm 0.008$ \\
\hline $56-3$ & $557.1 \pm 0.8$ & $0.459 \pm 0.006$ \\
\hline $56-4$ & $554 \pm 0.6$ & $0.44 \pm 0.01$ \\
\hline $56-5$ & $554.3 \pm 0.9$ & $0.459 \pm 0.007$ \\
\hline $56-6$ & $558.1 \pm 0.6$ & $0.451 \pm 0.01$ \\
\hline $56-7$ & $553 \pm 0.9$ & $0.445 \pm 0.008$ \\
\hline $56-8$ & $556.1 \pm 0.5$ & $0.456 \pm 0.01$ \\
\hline $56-9$ & $557.7 \pm 0.5$ & $0.463 \pm 0 ., 01$ \\
\hline $56-10$ & $555.2 \pm 1 ., 2$ & $0.46 \pm 0.008$ \\
\hline $56-11$ & $557.6 \pm 0.5$ & $0.469 \pm 0.002$ \\
\hline $56-12$ & $555.5 \pm 1.1$ & $0.458 \pm 0.006$ \\
\hline $56-13$ & $555 \pm 0.8$ & $0.469 \pm 0.004$ \\
\hline $\mathbf{5 6}$ & $\mathbf{5 5 5 . 8} \pm 2.0$ & $0.457 \pm 0.01$ \\
\hline
\end{tabular}

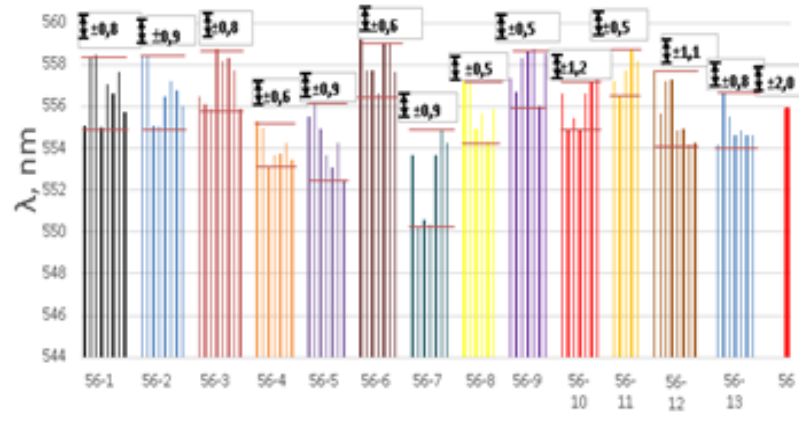

a)

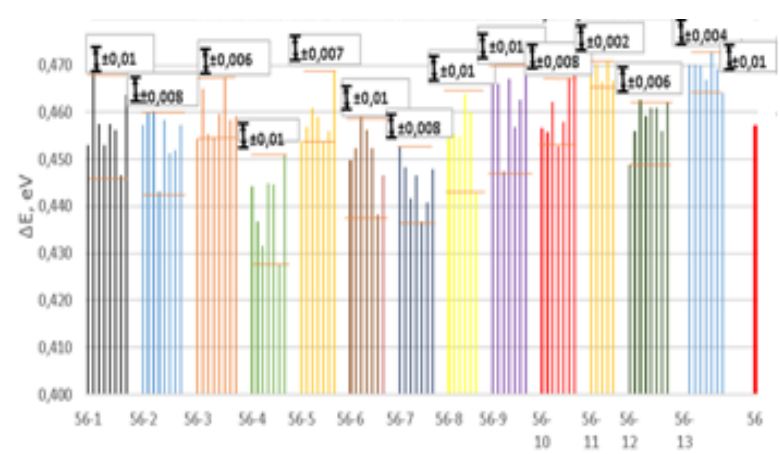

b)

Fig. 3. Dependence of band position (a) and band half-width (b) on the position in the crucible No.56

The same measurements were carried out for samples of series No.59. Photographs of the ceramic samples synthesized using a power density of $20 \mathrm{~kW} / \mathrm{cm} 2$ and their appearance after crushing in packages are shown in Figure 4. From the comparison of the photographs in Figures $1 \mathrm{a}$ and $\mathrm{b}$, it can be seen that the appearance of the synthesized samples 56 and 59 is quite different. The synthesis was carried out under the 
same irradiation conditions. The only difference was the difference in the yttrium oxide used for the synthesis while the stoichiometric composition of the initial charge was unchanged.

This difference also manifested itself in the bulk density value. The bulk density of the charge for sample 56 was $1.15 \mathrm{~g} / \mathrm{cm}^{3}$, while for sample 59 it was $1.05 \mathrm{~g} / \mathrm{cm}^{3}$. It should be emphasized that we cannot claim that the difference in bulk density in the mentioned intervals is determined by the bulk density value. As the experience of radiation synthesis of YAG:Ce ceramics has shown, there has never been a complete repetition of the samples during synthesis. The reason for this effect remains unknown.

The procedure for measuring the luminescence spectra of samples of series No. 59. When excited by radiation at $450 \mathrm{~nm}$ was completely similar to that described above. The results of the measurements are similar: the intensities are slightly different, the nature of the spectra is not. The results of the analysis are presented in Fig. 4, 5 and Table 2.A total of 7 samples were formed in crucible No.59. The measurements were carried out 10 times. Table 2 shows the average values of fringe position and half-width of the fringes with the indication of their error.

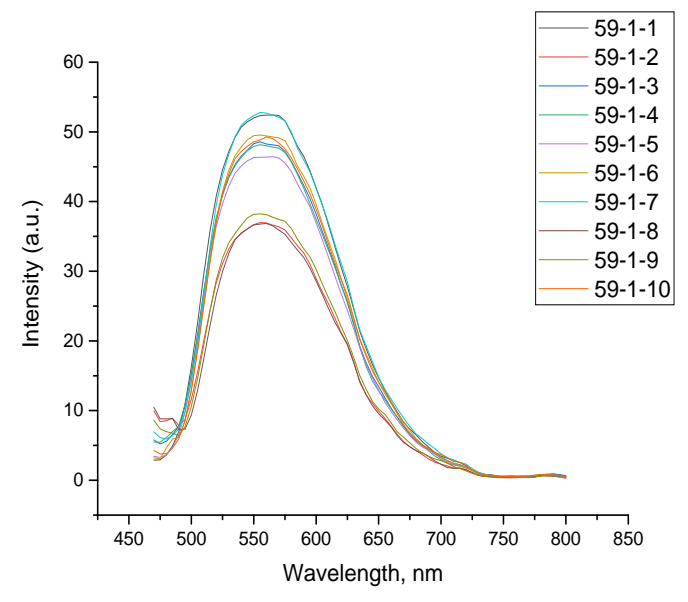

a)

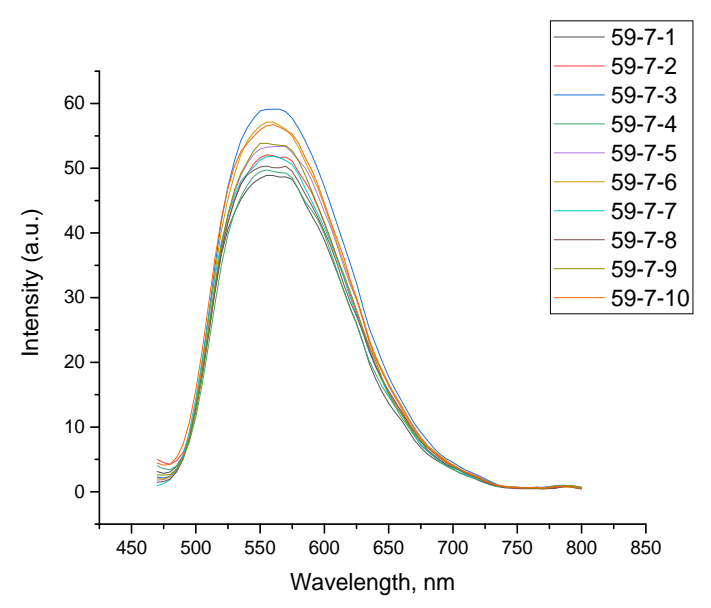

b)

Fig.4. Luminescence spectra of sample series: a) 59-1; b) 59-7.

Table 2. Mean values of the positions and half-widths of the bands and the values of measurement errors

\begin{tabular}{|c|c|c|}
\hline Sample name & $\boldsymbol{\lambda}_{\boldsymbol{s}}, \mathbf{n m}$ & $\mathbf{\Delta E}, \mathbf{e V}$ \\
\hline $59-1$ & $558.2 \pm 1.0$ & $0.456 \pm 0.01$ \\
\hline $59-2$ & $560 \pm 1.6$ & $0.465 \pm 0.005$ \\
\hline $59-3$ & $558.9 \pm 1.5$ & $0.459 \pm 0.006$ \\
\hline $59-4$ & $561.9 \pm 0.9$ & $0.469 \pm 0.008$ \\
\hline $59-5$ & $560.3 \pm 1.7$ & $0.456 \pm 0.009$ \\
\hline $59-6$ & $560.3 \pm 1.3$ & $0.459 \pm 0.007$ \\
\hline $59-7$ & $558.2 \pm 2.2$ & $0.453 \pm 0.01$ \\
\hline $\mathbf{5 9}$ & $\mathbf{5 5 9 . 6} \pm \mathbf{1 . 8}$ & $\mathbf{0 . 4 5 9} \pm \mathbf{0 . 0 0 8}$ \\
\hline
\end{tabular}

Figure 5 (a,b) also shows diagrams of the dependence of the fringe position (a) and fringe half-width (b) on the arrangement in the crucible. The crucible average of the band maximum is 559.6. The scatter of the band positions in the crucible is within 2-3 nm. The band half-width spread is 0.01-0.02 eV. Sample 59-7 has the largest scatter in the position of the bands than the other samples. 


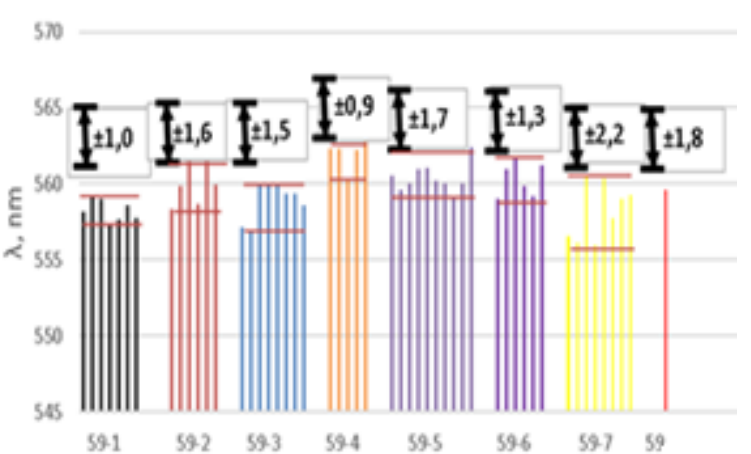

a)

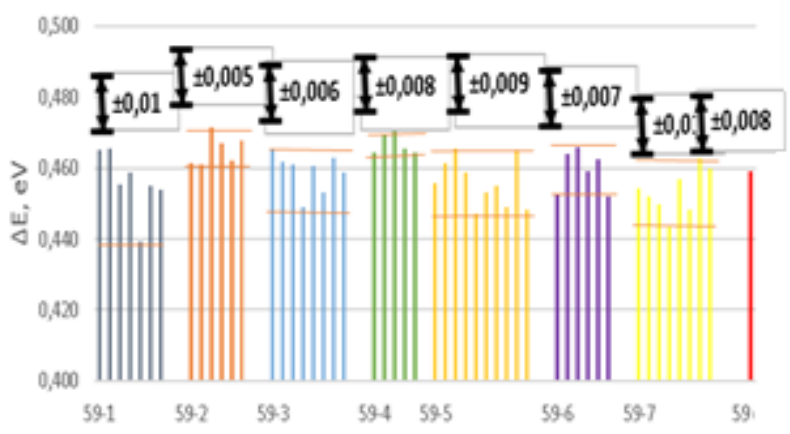

b)

Fig. 5. Dependence of band position (a) and band half-width (b) on the position in the crucible No. 59

\section{Conclusion}

Thus, in the field of a powerful flux of high-energy electrons, it is possible to synthesize ceramics based on YAG: Ce from a mixture of yttrium, aluminum, and cerium oxides with a predominant YAG structure. The type of ceramics obtained depends on the history of the reagents used. The spectral characteristics of the luminescence of YAG: Ce-based ceramic samples synthesized in the field of powerful radiation fluxes were measured, depending on the synthesis conditions. Were investigated samples of ceramics obtained at different times, with different compositions, different prehistories of the initial powders of oxides and the bulk density of the charge. In general, it can be seen that the scatter of the measured values of the characteristics of the bands in different samples of series 56 and 59 is approximately the same. The relative spread of the values of the positions of the bands and their half-widths in each individual sample can be greater than the average for all samples of the series. Thus, the change in the temperature regime during the formation of ceramics during scanning does not affect the scatter of the values of the characteristics of the bands in the samples.

The most probable reason for the scatter in the values of the spectral characteristics of luminescence may be that, at a high synthesis rate, a strained spatial structure is formed in crystallites due to the introduction of high concentrations of lattice defects. Distortion of the structure surrounded by luminescence centers, cerium ions, leads to deformation of the energy structure of the centers and, accordingly, their radiative properties.

\section{Acknowledgments}

The work was carried out within the framework of the grant AP08052050 of the Ministry of Education and Science of the Republic of Kazakhstan. This research was supported by Tomsk Polytechnic University CE Program.

\section{REFERENCES}

1 Michalik D., Sopicka-Lizer M., Plewa J., Pawlik T. Application of mechanochemical processing to synthesis of YAG:Ce garnet powder. Archives of metallurgy and materials. 2011, Vol. 56, pp.1258 - 1263.

2 Zehua Liu, Shuxing Li, et al. The effect of porosity on the $\mathrm{Al}_{2} \mathrm{O}_{3}$-YAG:Ce phosphor ceramic: Microstructure, luminescent efficiency, and luminous stability in laser driven lighting. Journal of Alloys and Compounds.2019, Vol. 785, pp.125-130.

3 Jian $\mathrm{Xu}$, Baofu $\mathrm{Hu}$, Chao Xu, et al. Carbon-free synthesis and luminescence saturation in a thick YAG:Ce film for laser-driven white lighting. Journal of the European Ceramic Society. 2019, Vol. 39, pp. 631-634.

4 Vaqueiro P., Lopez-Quintella M.A. Synthesis of yttrium-aluminium garnet by the citrate gel process. J.Mater. Chem.1998,Vol.8, No. 1, pp.161-163.

5 Davydova O.V., et al. Features of the synthesis of ultrafine powders of yttrium-aluminum garnet activated by cerium ions using the combustion method. Vestnik GSTU after P.O. Sukhoi. 2016, No. 2, pp.45-52.[in Russian].

6 Yadav P., Vijay K. Kumar Gupta, et al. One step combustion synthesis of YAG:Ce phosphor for solid state lighting. AIP Conference Proceedings. 2011, Vol.1391, pp. 200-202.

7 Ya-Wei Lee, Su-Hsen Wu. Fabrication and performance assessment of coprecipitation- based YAG:Cenanopowders for white LEDs.MicroelectronicEngineering.2018, Vol. 199, pp. 24-30.

8 Nien Y.T., Lu T.H., Bandi V.R., Chen I.G. Microstructure and photoluminescence characterizations of $\mathrm{Y}_{3} \mathrm{Al}_{5} \mathrm{O}_{12}:$ Ce phosphor ceramics sintered with silica. J. Am. Ceram. Soc.2012, Vol.95, pp.1378-1382.

9 Gyngazov S.A., Vasiliev I.P., Surzhikov A.P., et al. Ion treatment of zirconium ceramics with powerful pulsed beams. Journal of technical physics. 2015, Vol. 85, pp. 132 - 137.[in Russian].

10 Alekseev A.A., Mironovich A.Yu., Salogub D.V. Features of the Magnetic Structure of $\mathrm{Y}_{3} \mathrm{Fe}_{5} \mathrm{O}_{12}$ Polycrystals Synthesized by Radiation Thermal Sintering. Physics of the Solid State. 2020, Vol. 62, pp. 1156-1164. 
11 Kostishin V.G., Shakirzyanov R.I., Nalogin A.G., et al. Electrophysical and dielectric properties of polycrystals of yttrium iron garnet ferrite obtained by radiation-thermal sintering technology. Solid State Physics. 2021, Vol. 63, pp. 356 - 362. [in Russian].

12 Alekseev A.A., Mironovich A.Yu., Salogub D. V. Features of the Magnetic Structure of $\mathrm{Y}_{3} \mathrm{Fe}_{5} \mathrm{O}_{12}$ Polycrystals Synthesized by Radiation Thermal Sintering. Physics of the Solid State. 2020, Vol. 62, pp. 1156-1164.

13 Karipbaeyev Z., Polisadova E., Alpyssova G., et al. Dependence of the Efficiency Electron Beam Assisted Synthesis of YAG:Ce Ceramics on the Power Density of the Electron Flow. Proceedings of the $7^{\text {th }}$ International Congress on Energy Fluxes and Radiation Effects, EFRE 2020. 2020, pp. 892-895.

14 Lushchik Ch., Vitol IK, Elango MA Decay of electronic excitations into radiation defects in ionic crystals. Uspekhi fizicheskikh nauk. 1977, Vol. 122, no. 2, pp. 223-251.[in Russian].

15 Klinger I., Pushchin Ch.V., Mashovsts T.V., et al. Creation of defects in solids during decay of electronic excitations. Uspekhi fizicheskikh nauk. 1985, Vol.147, pp.523-558.[in Russian].

16 Elango, M.A. Elementary inelastic radiation processes.Nauka. 1988, 148 p.

17 Karipbayev Zh.T., Lisitsyn V.M., Mussakhanov D.A., Alpyssova G.K., et al. Time-resolved luminescence of YAG:Ce and YAGG:Ce ceramics prepared by electron beam assisted synthesis. Nuclear Instruments and Methods in Physics Research Section B: Beam Interactions with Materials and Atoms. 2020, Vol.479, pp. 222-228.

18 Lisitsyn V.M., Lisitsyna L.A., Golkovsky M.G., et al. Formation of luminescent high-temperature ceramics in a powerful flow of high-energy electrons. Izvestiya VUZov. 2020,No. 9, pp.102-107 [in Russian].

19 Alpyssova G., Mussakhanov D., Karipbayev Zh., et al. Luminescence spectra of YAG:Ce phosphors synthesized in a field of radiation. IOP Conf. Series: Materials Science and Engineering. 2020, No.754, pp. 012014.

20 Mussakhanov D.A., Tulegenova A.T., Lisitsyn V.M., et al. Structural and luminescent characteristics of YAG phosphors synthesized in the radiation field. IOP Conference Series: Materials Science and Engineering. 2019 , Vol. 510, Issue 1, pp. 012031.

21 Karipbaev Zh., Musakhanov D., Lisitsyn V., Alpyssova G., et al. Synthesis, the study of the structure of YAG and YAGG phosphors in the radiation field. Bulletin of Karaganda University. Physics series. 2019, No. 4(96), pp. 24 -29 .

22 Karipbayev Zh., Alpyssova G., Mussakhanov D., Lisitsyn V., Kukenova A., Tulegenova A. Time-resolved luminescence excited with $\mathrm{N}_{2}$ laser of YAG:Ce Ceramics formed by electron beam assisted synthesis. Eurasian Physical Technical Journal. 2020, Vol.17, No.1(33), pp.73- 76. 